\title{
Genetic evidence for recent and incipient speciation in the evolution of Hawailan Metrosideros (Myrtaceae)
}

\author{
K. M. ARADHYA, D. MUELLER-DOMBOIS \& T. A. RANKER* \\ Department of Botany, University of Hawaii at Manoa, Honolulu, HI 96822, and *University Museum, Campus Box 350 , \\ University of Colorado, Boulder, CO 80309 , USA
}

\begin{abstract}
The genus Metrosideros Banks in Hawaii comprises five morphologically recognizable species. In the present study $M$. rugosa, $M$. tremuloides, and $M$. polymorpha were analysed for isozyme variation using starch gel electrophoresis. Fourteen populations from the island of Oahu were assessed for four enzymes encoded by six polymorphic loci. The populations exhibited high levels of variation with mean numbers of alleles per locus ranging from 2.30 to 3.30 with an overall mean of 2.97. The mean observed populational heterozygosity for different populations varied from 0.100 to 0.368 with a mean of 0.272 . Gene diversity analysis indicated that 87.7 per cent of the total genetic variation resides within populations, while only 4.7 per cent was due to differentiation among taxa. The mean unbiased genetic identity for pairwise comparisons of species was 0.904 , which is very high for congeneric flowering plant species. Therefore, in spite of distinct morphological divergence, the Metrosideros taxa studied did not show expected levels of divergence at genes coding for the enzymes studied. The data suggest that both $M$. rugosa and $M$. tremuloides have diverged recently from $M$. polymorpha. However, $M$. rugosa has diverged more $(\bar{I}=0.876)$ than $M$. tremuloides $(\bar{I}=0.917)$. This may therefore represent a case of recent and incipient speciation in the insular environment.
\end{abstract}

Keywords: evolution, genetic differentiation, incipient speciation, isozymes, Metrosideros.

\section{Introduction}

Most continental biota are so ancient that the key events of evolution that formed them have been lost (Carson, 1987). In contrast to this, island biota provide excellent opportunities for biologists to study the dynamic processes of evolution and speciation. The Hawaiian archipelago is a highly isolated group of volcanic islands in the pacific situated $4000 \mathrm{~km}$ from the nearest continent. Colonization processes have populated the windward slopes of the volcanoes with forest ecosystems up to altitudes of about $2000 \mathrm{~m}$. Climatic and edaphic factors vary dramatically over short distances in these islands, providing numerous niches to which plants may become adapted. This ecological diversity has been accompanied by the evolution of literally hundreds of species unique to these islands (Carson, 1987). Mueller-Dombois (1981) aptly describes the Hawaiian biota as naturally depauperated and secondarily enriched. However, the high rate of evolution and speciation in these islands is also accompanied by an increased rate of extinction due to natural and anthropogenic habitat modification.

The genus Metrosideros Banks (Myrtaceae) is widely distributed in the six major islands of the Hawaiian Archipelago (i.e. Kauai, Oahu, Molokai, Lanai, Maui, and Hawaii). This genus also occurs naturally in the other oceanic islands of the Pacific from about $52^{\circ} \mathrm{S}$ latitude on Auckland Island to about $25^{\circ} \mathrm{N}$ latitude and from Australia and Malaysia in the west to French Polynesia in the east (Smith, 1973). Metrosideros comprises an abundant and taxonomically confusing species complex throughout much of its range (Smith, 1973). At least five species have been described from Hawaii entirely based on morphological criteria (Rock, 1917; Skottsberg, 1944). Metrosideros polymorpha is the dominant tree species in Hawaii and occupies over 80 per cent of the relatively intact forests (MuellerDombois \& Loope, 1990). The remaining four species; $M$. macropus, $M$. rugosa, $M$. tremuloides and $M$. 
waialealae, are of much narrower distribution and occur sympatrically with $M$. polymorpha on the island of Oahu.

Metrosideros polymorpha has an extremely wide ecological amplitude that occurs from near sea level to the tree line at $2500 \mathrm{~m}$ on Mauna Loa on the island of Hawaii. It is well adapted to cloud forest conditions where it can grow to $20 \mathrm{~m}$ tall. On exposed, eroded ridges it occurs as a shrub or low growing tree and in bogs grows as a prostrate shrub or small tree, sometimes flowering when under $10 \mathrm{~cm}$ tall. Metrosideros polymorpha is the first tree to colonize new lava flows where it forms mature, closed stands in about 400 years in the rainforest climate (Atkinson, 1970).

Metrosideros polymorpha exhibits very high levels of morphological diversity and adaptation in the Hawaiian islands. Rock (1917) identified 11 varieties in an attempt to classify the variability within $M$. polymorpha and Porter (1972) treated all taxa of Metrosideros as varieties and forms of this species. Corn (1979), however, reported clinal morphological variation in Hawaiian Metrosideros correlated with a complex altitudinal gradient, and Stemmermann (1983) discovered a positive association of morphological variation with age of substrate. The validity of the described infraspecific taxa is therefore, questionable.

Three morphologically distinct taxa, $M$. rugosa, $M$. tremuloides and $M$. polymorpha, from the Koolau and Waianae mountains of the island of Oahu, were involved in the present study. Metrosideros rugosa is a shrub or small tree mainly restricted to dry, eroded ridge tops in the Koolaus, characterized by strongly rugose leaves with lower surfaces densely woolly. Metrosideros tremuloides is also a shrub or low growing tree with a semi-weeping habit and occurring on steep slopes of the Koolau and Waianae mountains. The leaves are distinctively small and narrow. Metrosideros polymorpha is highly variable in size, ranging froshrubs to tall trees, inhabiting a wide range of habita . The leaf shape and flower colour are also highly variable.

The information on the breeding system in these taxa is lacking. However, an earlier study has shown that these taxa have different peak flushing and flowering periods but the temporal isolation is incomplete (Porter, 1972). Corn (1979) reported that there is extensive outcrossing in Metrosideros because the flowers are protandrous with sticky pollen that is transmitted by native birds that visit the flowers for nectar. Carpenter (1976) observed partial self-incompatibility among red-flowered types and normal seed-set occurs only with outbreeding. The presence of morphologically intermediate forms of these taxa in natural populations may also indicate that these taxa are still connected through significant levels of gene flow. Metrosideros bear numerous and small seeds, but only about 15 per cent of the seeds appeared to be well filled with embryos and about 30 per cent germinate (Burton, 1982). Wind dispersal is probably the chief seed dispersal mechanism of this genus (Corn, 1972).

The extensive variability exhibited by the Hawaiian Metrosideros complex, involving either closely related species and/or varieties within species, offers a unique opportunity to study the evolutionary mechanisms that operate in plant populations. Morphological studies alone are often inadequate to determine evolutionary relationships in such complexes (Systma \& Schaal, 1985). Hence, integrative studies involving variation for biochemical characters such as isozymes or other macromolecules, in addition to morphological comparisons, are most effective in such diverse groups.

The purpose of this study was to

1 assess genetic variation in the Hawaiian species complex of the genus Metrosideros;

2 determine the extent of genetic divergence among morphologically discrete taxa treated as different species; and

3 re-evaluate these taxa systematically in the light of genetic evidence.

\section{Materials and methods}

Three populations of $M$. rugosa, two of $M$. tremuloides, and nine of $M$. polymorpha were sampled on the island of Oahu, Hawaii (Table 1 and Fig. 1). A minimum of 30 sexually mature trees were sampled per population for fresh young leaves. The samples were transported to the laboratory on ice, stored at $4^{\circ} \mathrm{C}$, and analysed within 7 days.

\section{Electrophoresis}

Leaf samples were homogenized in a buffer containing 10 per cent dimethyl sulphoxide (DMSO), $0.02 \mathrm{M}$ sodium metabisulphate, and $0.005 \mathrm{M}$ diethyldithiocarbomate (DIECA) in addition to the other constituents listed by Bousquet et al. (1987). The homogenate was absorbed onto Whatman No. 3 filterpaper wicks $(3 \mathrm{~mm} \times 1.0 \mathrm{~cm})$ and loaded onto $12 \mathrm{per}$ cent starch gels prepared with a histidine-citrate buffer at pH 6.5 (Cardy et al., 1983). The gel buffer consisted of $0.016 \mathrm{~m}$ histidine (free base) and $0.002 \mathrm{M}$ citric acid and the tray buffer of $0.065 \mathrm{M}$ histidine and $0.007 \mathrm{M}$ citric acid. Gels were cooled to $4^{\circ} \mathrm{C}$ before loading samples. Electrophoresis was conducted in a refrigerator at $4^{\circ} \mathrm{C}$ and at $200 \mathrm{~V}\left(20 \mathrm{~V} \mathrm{~cm}^{-1}\right)$ with $40 \mathrm{~mA}$ for $6 \mathrm{~h}$. Four enzyme systems, known to be polymorphic (PGI, PGM, PER, and LAP; K.M. Aradhya, 
Table 1 Populations and species of Metrosideros studied

\begin{tabular}{|c|c|c|c|}
\hline $\begin{array}{l}\text { Population } \\
\text { designation }\end{array}$ & $\begin{array}{l}\text { Altitude } \\
\text { (m) }\end{array}$ & Taxon & $\begin{array}{l}\text { Approximate } \\
\text { location }\end{array}$ \\
\hline 1 (MR,PU-1) & 550 & M. rugosa & $\begin{array}{l}\text { Off Koolau ridge summit } \\
\text { trail, Pupukea, Koolauloa } \\
\text { District }\end{array}$ \\
\hline 2 (MR,PU-2) & 570 & M. rugosa & $\begin{array}{l}\text { About } 0.5 \mathrm{~km} \text { away from } \\
\text { MR,PU-1 along ridge trail }\end{array}$ \\
\hline $3(\mathrm{MR}, \mathrm{P})$ & 600 & M. rugosa & $\begin{array}{l}\text { Pauoa, above Pauoa flats, } \\
\text { off Tantalus trail, along } \\
\text { summit }\end{array}$ \\
\hline $4(\mathrm{MT}, \mathrm{P})$ & 570 & M. tremuloides & $\begin{array}{l}\text { Pauoa, above Pauoa flats, } \\
\text { off Tantalus trail, } \\
\text { Honolulu District }\end{array}$ \\
\hline 5 (MT,PS) & 570 & M. tremuloides & $\begin{array}{l}\text { Pauoa, above Pauoa flats, } \\
\text { off Tantalus trail, along } \\
\text { summit }\end{array}$ \\
\hline $6(\mathrm{MP}, \mathrm{PU})$ & 570 & M. polymorpha & $\begin{array}{l}\text { Above Pauoa flats, along } \\
\text { summit, Honolulu District }\end{array}$ \\
\hline 7 (MP,WK) & 650 & M. polymorpha & $\begin{array}{l}\text { Northern ridge of Waianae } \\
\text { Kai, Waianae District }\end{array}$ \\
\hline 8 (MP,PK,EWA) & 750 & M. polymorpha & $\begin{array}{l}\text { Puu Kaua, Ewa side slope, } \\
\text { Ewa District }\end{array}$ \\
\hline 9 (MP,PK,WS) & 700 & M. polymorpha & $\begin{array}{l}\text { Puu Kaua, Waianae side } \\
\text { slope, Waianae District }\end{array}$ \\
\hline 10 (MP,ML) & 475 & M. polymorpha & $\begin{array}{l}\text { Mauumae ridge summit } \\
\text { trail above Maunalani } \\
\text { heights, Honolulu District }\end{array}$ \\
\hline 11 (MP,AH-1) & 400 & M. polymorpha & $\begin{array}{l}\text { Aiea Heights loop trail, } \\
\text { above Aiea Heights Park. } \\
\text { Ewa District }\end{array}$ \\
\hline 12 (MP,AH-2) & 425 & M. polymorpha & $\begin{array}{l}\text { Aiea Heights loop trail } \\
\text { towards Kalauao ridge }\end{array}$ \\
\hline 13 (MP,AH-3) & 425 & M. polymorpha & $\begin{array}{l}\text { Aiea Heights trail } \\
\text { towards Kalauao ridge }\end{array}$ \\
\hline 14 (MP,AH-4) & 400 & M. polymorpha & $\begin{array}{l}\text { Aiea Heights trail towards } \\
\text { Kalauao ridge }\end{array}$ \\
\hline
\end{tabular}

unpublished data) were assayed following Arulsekar \& Parfitt (1986) and Shaw \& Prasad (1970).

\section{Data analysis}

Genotype frequencies were inferred directly from observed isozyme phenotypes. The mean number of alleles per locus, observed and expected levels of heterozygosity and Nei's unbiased genetic identity and distance coefficients (Nei, 1978) were computed with the BIOsYs program (Swofford \& Selander, 1989).

The genetic diversity within the Metrosideros species complex was partitioned using the gene diversity analysis (Nei, 1973). Total gene diversity $\left(H_{\mathrm{T}}\right)$ was partitioned into gene diversity within populations $\left(H_{\mathrm{S}}\right)$ and gene diversity among populations $\left(D_{\mathrm{ST}}\right)$ where $H_{\mathrm{T}}=H_{\mathrm{S}}+D_{\mathrm{ST}} \cdot \mathrm{H}_{\mathrm{T}}$ was calculated on the weighted average allele frequencies over all populations 


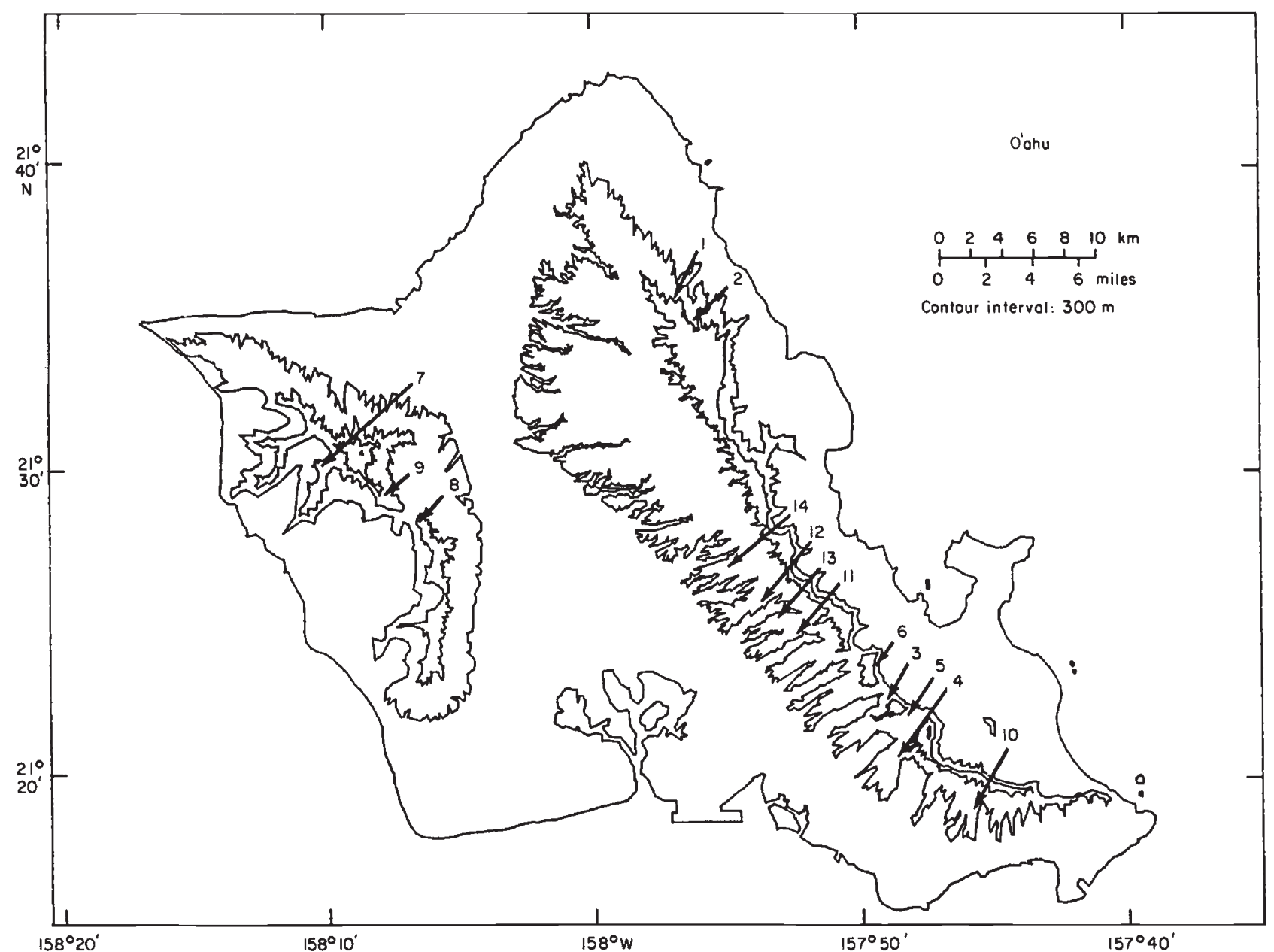

Fig. 1 Approximate location of collection sites for 14 populations of Metrosideros.

$\left(H_{\mathrm{T}}=1-\Sigma \bar{p}_{\mathrm{i}}^{2}\right) . H_{\mathrm{S}}$ is equal to the weighted average over all populations of the values of $1-\Sigma p_{i}^{2}$ for each population. $D_{\mathrm{ST}}$ is obtained by subtraction $\left(D_{\mathrm{ST}}=H_{\mathrm{T}}-H_{\mathrm{S}}\right)$. Differentiation among populations is calculated as $G_{\mathrm{ST}}=D_{\mathrm{ST}} / H_{\mathrm{T}}$ ) where $G_{\mathrm{ST}}$ can vary between 0 (when $H_{\mathrm{S}}=H_{\mathrm{T}}$ ) and 1 (when $H_{\mathrm{S}}=0$ ), i.e. populations fixed for different alleles.

Similarly specieswise pooled analysis was performed to further partition $G_{\mathrm{ST}}$ into gene diversity due to among populations within taxa and among taxa. It was decided not to pool the data across loci as the rate of divergence was different.

A UPGMA cluster analysis was performed on populational, pairwise genetic identity coefficients (Sneath \& Sokal, 1973).

\section{Results}

\section{Genetic variability}

The six putative loci assayed were Pgi, Pgm-1, Pgm-2, Per, Lap-1 and Lap-2. The allelic frequencies in 14 populations are given in Table 2. A total of 30 alleles were detected, with the number of alleles per locus ranging from three for $L a p-2$ to eight for Pgm-1. The patterns of allelic distribution and number of alleles per locus varied across populations. For most loci, there was at least one common allele at high frequency in most populations. Two alleles $(87$ and 100$)$ were equally predominant in most populations for $\mathrm{Per}$, and allele 83 was unique to population 3 (Table 1 ) of $M$. rugosa. The Waianae populations of $M$. polymorpha (7-9) possessed five unique, low frequency alleles at Pgi, Pgm-1 and Pgm-2. Metrosideros polymorpha populations from Aiea (11-14) did not possess allele 110 for Lap-2, which was expressed in all other populations. All alleles expressed in $M$. tremuloides populations ( 4 and 5) were a subset of those observed in $M$. polymorpha and these two species uniquely shared Lap-1 allele 89.

The intra-populational estimates of genetic variability are presented in Table 3 . The mean number of alleles per locus ranged from 2.3 to 3.3 with a mean of 3.0. The mean for $M$. rugosa populations was the 
Table 2 Allelic frequencies for the six polymorphic loci in Hawaiian Metrosideros

Population

\begin{tabular}{|c|c|c|c|c|c|c|c|c|c|c|c|c|c|c|}
\hline cus & 1 & 2 & 3 & 4 & 5 & 6 & 7 & 8 & 9 & 10 & 11 & 12 & 13 & 14 \\
\hline \multicolumn{15}{|l|}{$\theta^{\circ}$} \\
\hline$(\mathrm{N})$ & 40 & 36 & 45 & 35 & 40 & 40 & 50 & 38 & 35 & 35 & 30 & 30 & 30 & 30 \\
\hline 88 & 0.025 & 0.028 & 0.000 & 0.000 & 0.000 & 0.025 & 0.0 & 0.0 & 0.0 & 0.0 & 0.0 & 0.000 & 0. & \\
\hline 9 & 0.013 & 0 & 0 & 0.000 & 0.0 & 0.0 & $0 .($ & 0.0 & 0.0 & 0.0 & 0. & 0 & 0. & \\
\hline 100 & 0.925 & 0.9 & 0.967 & 0.800 & 0.9 & 0.9 & 0.7 & 0.566 & 0.671 & 0.7 & 0. & 33 & 0.850 & 0.817 \\
\hline 108 & 0.000 & 0.000 & 0.022 & 0.186 & 0.075 & 0.000 & 0.000 & 0.000 & 0.000 & 0.043 & 0.083 & 0.167 & 0.083 & 0.150 \\
\hline 112 & 0.038 & 0.042 & 0.011 & 0.014 & 0.000 & 0.025 & 0.240 & 0.421 & 0.314 & 0.200 & 0.000 & 0.000 & 0.000 & 0.000 \\
\hline 122 & 0.000 & 0.000 & 0.000 & 0.000 & 0.000 & 0.000 & 0.010 & 0.013 & 0.014 & 0.000 & 0.000 & 0.000 & 0.000 & 0.000 \\
\hline
\end{tabular}

Pgm-1

$\begin{array}{rllllllllllllllll}(\mathrm{N}) & 40 & 40 & 45 & 35 & 40 & 40 & 40 & 38 & 39 & 35 & 30 & 30 & 30 & 30 \\ 60 & 0.000 & 0.000 & 0.000 & 0.000 & 0.000 & 0.000 & 0.000 & 0.000 & 0.026 & 0.000 & 0.000 & 0.000 & 0.000 & 0.000 \\ 86 & 0.000 & 0.000 & 0.000 & 0.000 & 0.000 & 0.000 & 0.000 & 0.092 & 0.077 & 0.000 & 0.000 & 0.000 & 0.000 & 0.000 \\ 91 & 0.025 & 0.025 & 0.022 & 0.000 & 0.000 & 0.013 & 0.000 & 0.171 & 0.115 & 0.014 & 0.067 & 0.000 & 0.067 & 0.017 \\ 95 & 0.175 & 0.175 & 0.122 & 0.143 & 0.038 & 0.100 & 0.175 & 0.145 & 0.231 & 0.114 & 0.067 & 0.000 & 0.017 & 0.050 \\ 100 & 0.425 & 0.488 & 0.422 & 0.543 & 0.950 & 0.750 & 0.738 & 0.447 & 0.423 & 0.786 & 0.783 & 0.883 & 0.550 & 0.617 \\ 109 & 0.063 & 0.087 & 0.111 & 0.214 & 0.013 & 0.087 & 0.038 & 0.105 & 0.038 & 0.014 & 0.083 & 0.117 & 0.217 & 0.200 \\ 118 & 0.313 & 0.225 & 0.311 & 0.100 & 0.000 & 0.050 & 0.050 & 0.039 & 0.051 & 0.071 & 0.000 & 0.000 & 0.150 & 0.117 \\ 123 & 0.000 & 0.000 & 0.011 & 0.000 & 0.000 & 0.000 & 0.000 & 0.000 & 0.038 & 0.000 & 0.000 & 0.000 & 0.000 & 0.000\end{array}$

$P g m-2$

$\begin{array}{rlllllllllllllllll}(\mathrm{N}) & 40 & 40 & 45 & 35 & 40 & 40 & 40 & 38 & 37 & 35 & 30 & 30 & 30 & 30 \\ 80 & 0.000 & 0.000 & 0.000 & 0.000 & 0.000 & 0.000 & 0.000 & 0.013 & 0.000 & 0.000 & 0.000 & 0.000 & 0.000 & 0.000 \\ 95 & 0.138 & 0.038 & 0.067 & 0.086 & 0.000 & 0.013 & 0.038 & 0.118 & 0.135 & 0.057 & 0.033 & 0.083 & 0.000 & 0.000 \\ 100 & 0.700 & 0.762 & 0.600 & 0.829 & 1.000 & 0.938 & 0.863 & 0.789 & 0.703 & 0.943 & 0.967 & 0.917 & 0.917 & 0.900 \\ 105 & 0.162 & 0.200 & 0.333 & 0.086 & 0.000 & 0.050 & 0.075 & 0.079 & 0.162 & 0.000 & 0.000 & 0.000 & 0.083 & 0.100 \\ 109 & 0.000 & 0.000 & 0.000 & 0.000 & 0.000 & 0.000 & 0.025 & 0.000 & 0.000 & 0.000 & 0.000 & 0.000 & 0.000 & 0.000\end{array}$
Per

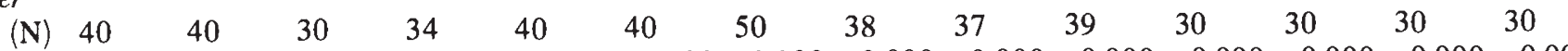

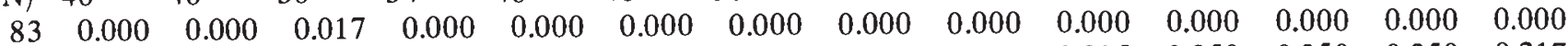

$\begin{array}{lllllllllllllll}87 & 0.450 & 0.587 & 0.067 & 0.338 & 0.175 & 0.363 & 0.060 & 0.118 & 0.149 & 0.295 & 0.250 & 0.350 & 0.350 & 0.217\end{array}$

$\begin{array}{llllllllllllllll}100 & 0.538 & 0.412 & 0.917 & 0.662 & 0.825 & 0.637 & 0.510 & 0.882 & 0.851 & 0.705 & 0.750 & 0.633 & 0.617 & 0.783\end{array}$

$\begin{array}{lllllllllllllll}130 & 0.013 & 0.000 & 0.000 & 0.000 & 0.000 & 0.000 & 0.430 & 0.000 & 0.000 & 0.000 & 0.000 & 0.017 & 0.033 & 0.000\end{array}$

\begin{tabular}{|c|c|c|c|c|c|c|c|c|c|c|c|c|c|c|}
\hline \multicolumn{15}{|l|}{ Lap-1 } \\
\hline & 40 & 40 & 12 & 35 & 40 & 40 & 40 & 38 & 37 & 35 & 30 & 30 & 30 & 30 \\
\hline 89 & 0.000 & 0.000 & 0.000 & 0.129 & 0.075 & 0.000 & 0.000 & 0.000 & 0.000 & 0.157 & 0.133 & 0.067 & 0.000 & 0.000 \\
\hline 95 & 0.225 & 0.287 & 0.125 & 0.257 & 0.100 & 0.162 & 0.237 & 0.158 & 0.162 & 0.157 & 0.150 & 0.067 & 0.317 & 0.300 \\
\hline 100 & 0.300 & 0.587 & 0.458 & 0.586 & 0.825 & 0.688 & 0.738 & 0.842 & 0.838 & 0.686 & 0.717 & 0.867 & 0.683 & 0.700 \\
\hline 105 & 0.475 & 0.125 & 0.417 & 0.029 & 0.000 & 0.150 & 0.025 & 0.000 & 0.000 & 0.000 & 0.000 & 0.000 & 0.000 & 0.000 \\
\hline \multicolumn{15}{|l|}{ Lap-2 } \\
\hline (N) & 40 & 40 & 12 & 35 & 40 & 40 & 40 & 38 & 37 & 35 & 30 & 30 & 30 & 30 \\
\hline 97 & 0.000 & 0.150 & 0.000 & 0.000 & 0.075 & 0.050 & 0.000 & 0.000 & 0.000 & 0.000 & 33 & 33 & 0.383 & 0.250 \\
\hline 100 & 0.438 & 0.475 & 0.542 & 0.971 & 0.800 & 0.637 & 0.613 & 0.842 & 0.946 & 71 & 0. & 0.867 & 0.617 & 0.750 \\
\hline 110 & 0.563 & 0.375 & 0.458 & 0.029 & 0.125 & 0.313 & 0.387 & 0.158 & 0.054 & 0.329 & 0.000 & 0.000 & 0.000 & 0.000 \\
\hline
\end{tabular}

highest (3.3) followed by the populations of $M$. polymorpha (2.9) and $M$. tremuloides (2.7). Mean observed heterozygosity ranged from 0.100 to 0.368 with an overall mean of 0.272 . Mean observed heterozygosity was the highest for $M$. rugosa $(0.323)$ followed by $M$. polymorpha $(0.269)$ and $M$. tremuloides $(0.208)$. The expected heterozygosity followed the same trend with the highest recorded for $M$. rugosa $(0.470)$ followed by $M$. polymorpha $(0.360)$ and $M$. tremuloides $(0.296)$.

The fixation index ( $F$; Wright, 1965; Jain \& Workman, 1967) was calculated for all populations and averaged across loci (Table 3 ). The index is equal to 
Table 3 Genetic variability in Hawaiian Metrosideros (standard errors in parentheses)

\begin{tabular}{|c|c|c|c|c|c|}
\hline \multirow[b]{2}{*}{$\begin{array}{l}\text { Population } \\
\text { designation }\end{array}$} & \multirow[b]{2}{*}{ Taxon } & \multirow{2}{*}{$\begin{array}{l}\text { Mean number } \\
\text { of alleles } \\
\text { per locus }\end{array}$} & \multirow[b]{2}{*}{$F^{*}$} & \multicolumn{2}{|c|}{ Mean heterozygosity } \\
\hline & & & & $\begin{array}{l}\text { Direct- } \\
\text { count }\end{array}$ & $\begin{array}{l}\text { HdyWbg } \\
\text { expected } \dagger\end{array}$ \\
\hline 1. & M. rugosa & $\begin{array}{c}3.3 \\
(0.4)\end{array}$ & $0.265 \ddagger$ & $\begin{array}{c}0.363 \\
(0.089)\end{array}$ & $\begin{array}{c}0.494 \\
(0.079)\end{array}$ \\
\hline 2. & M. rugosa & $\begin{array}{c}3.3 \\
(0.4)\end{array}$ & $0.340 \ddagger$ & $\begin{array}{c}0.319 \\
(0.097)\end{array}$ & $\begin{array}{c}0.483 \\
(0.077)\end{array}$ \\
\hline 3. & M. rugosa & $\begin{array}{c}3.3 \\
(0.6)\end{array}$ & $0.341 \ddagger$ & $\begin{array}{c}0.286 \\
(0.097)\end{array}$ & $\begin{array}{c}0.434 \\
(0.106)\end{array}$ \\
\hline 4. & M. tremuloides & $\begin{array}{c}3.0 \\
(0.4)\end{array}$ & 0.198 & $\begin{array}{c}0.316 \\
(0.059)\end{array}$ & $\begin{array}{c}0.394 \\
(0.087)\end{array}$ \\
\hline 5. & M. tremuloides & $\begin{array}{c}2.3 \\
(0.3)\end{array}$ & $0.492 \ddagger$ & $\begin{array}{c}0.100 \\
(0.047)\end{array}$ & $\begin{array}{c}0.197 \\
(0.056)\end{array}$ \\
\hline 6. & M. polymorpha & $\begin{array}{c}3.2 \\
(0.4)\end{array}$ & $0.438 \ddagger$ & $\begin{array}{c}0.196 \\
(0.056)\end{array}$ & $\begin{array}{c}0.349 \\
(0.077)\end{array}$ \\
\hline 7. & M. polymorpha & $\begin{array}{c}3.2 \\
(0.3)\end{array}$ & $0.252 \ddagger$ & $\begin{array}{c}0.312 \\
(0.050)\end{array}$ & $\begin{array}{c}0.417 \\
(0.042)\end{array}$ \\
\hline 8. & M. polymorpha & $\begin{array}{c}3.2 \\
(0.7)\end{array}$ & 0.064 & $\begin{array}{c}0.368 \\
(0.091)\end{array}$ & $\begin{array}{c}0.393 \\
(0.081)\end{array}$ \\
\hline 9. & M. polymorpha & $\begin{array}{c}3.3 \\
(1.0)\end{array}$ & $0.255 \ddagger$ & $\begin{array}{c}0.287 \\
(0.087)\end{array}$ & $\begin{array}{c}0.385 \\
(0.092)\end{array}$ \\
\hline 10 & M. polymorpha & $\begin{array}{c}2.8 \\
(0.5)\end{array}$ & $0.132 \ddagger$ & $\begin{array}{c}0.322 \\
(0.068)\end{array}$ & $\begin{array}{c}0.371 \\
(0.055)\end{array}$ \\
\hline 11. & M. polymorpha & $\begin{array}{c}2.7 \\
(0.3)\end{array}$ & $0.208 \ddagger$ & $\begin{array}{c}0.206 \\
(0.081)\end{array}$ & $\begin{array}{c}0.260 \\
(0.069)\end{array}$ \\
\hline 12. & M. polymorpha & $\begin{array}{c}2.5 \\
(0.2)\end{array}$ & $0.429 \ddagger$ & $\begin{array}{c}0.161 \\
(0.048)\end{array}$ & $\begin{array}{c}0.282 \\
(0.049)\end{array}$ \\
\hline 13. & M. polymorpha & $\begin{array}{c}2.8 \\
(0.5)\end{array}$ & 0.222 & $\begin{array}{c}0.322 \\
(0.056)\end{array}$ & $\begin{array}{c}0.414 \\
(0.071)\end{array}$ \\
\hline 14. & M. polymorpha & $\begin{array}{c}2.7 \\
(0.5)\end{array}$ & $0.326 \ddagger$ & $\begin{array}{c}0.250 \\
(0.041)\end{array}$ & $\begin{array}{c}0.371 \\
(0.053)\end{array}$ \\
\hline Mean & & 3.0 & $0.283 \ddagger$ & 0.272 & 0.371 \\
\hline
\end{tabular}

*Fixation index (Wright, 1965).

†Unbiased estimate (see Nei, 1978).

$\ddagger P<0.05$.

$\left(H_{\exp }-H_{\text {obs }}\right) / H_{\text {exp }}$, where $H_{\text {exp }}$ and $H_{\text {obs }}$ refer to expected and observed heterozygosities, respectively. There was a significant deficiency of heterozygotes as compared to Hardy-Weinberg expectations in most populations except for populations 4 ( $M$. tremuloides), 8 and 13 (both $M$. polymorpha) $(P<0.05)$.

\section{Population differentiation}

The gene diversity analysis of the Metrosideros complex (Table 4) indicated that the total gene diversity $\left(H_{\mathrm{T}}\right)$, a measure of mean heterozygosity expected under random mating, ranged from 0.287 for $P g m-2$ to 0.578 for $P g m-1$ with an overall mean of 0.423 . The intrapopulational gene diversity summed over all loci accounted for a significant proportion of the total gene diversity ( 87.7 per cent), while diversity due to differentiation among populations $\left(G_{\mathrm{ST}}\right)$ ranged from 9.1 per cent for Pgm-2 to 18.1 per cent for Lap-2 with an average of 12.3 per cent. Further partitioning of the $G_{\mathrm{ST}}$ component into that among populations within taxa and that among taxa indicated that a greater proportion of gene diversity due to interpopulational differentiation resides among populations within taxa (7.6 per cent) compared to among taxa ( 4.8 per cent).

The unbiased genetic distance $(D)$ and identity $(I)$ measures of Nei (1978) were calculated for both pairwise comparisons among the 14 populations and 
Table 4 Measures of gene diversity and population differentiation in Metrosideros

\begin{tabular}{|c|c|c|c|c|c|c|c|}
\hline \multicolumn{5}{|c|}{ Differentiation among populations } & \multicolumn{3}{|c|}{ Apportionment of diversity } \\
\hline Locus & $H_{\mathrm{T}}$ & $H_{\mathrm{S}}$ & $D_{\mathrm{ST}}$ & $G_{\mathrm{ST}}$ & $\begin{array}{l}\text { Within } \\
\text { populations }\end{array}$ & $\begin{array}{l}\text { Among populations } \\
\text { within taxa }\end{array}$ & $\begin{array}{l}\text { Among } \\
\text { taxa }\end{array}$ \\
\hline$P g i$ & 0.302 & 0.267 & 0.035 & 0.116 & 0.884 & 0.083 & 0.033 \\
\hline Pgm-1 & 0.578 & 0.522 & 0.056 & 0.097 & 0.903 & 0.057 & 0.040 \\
\hline Pgm-2 & 0.287 & 0.261 & 0.026 & 0.091 & 0.909 & 0.042 & 0.049 \\
\hline Per & 0.457 & 0.392 & 0.065 & 0.142 & 0.858 & 0.125 & 0.018 \\
\hline Lap-1 & 0.482 & 0.430 & 0.052 & 0.108 & 0.892 & 0.048 & 0.060 \\
\hline Lap-2 & 0.430 & 0.352 & 0.078 & 0.181 & 0.819 & 0.095 & 0.086 \\
\hline Mean & 0.423 & 0.371 & 0.052 & 0.123 & 0.877 & 0.076 & 0.047 \\
\hline
\end{tabular}

$H_{\mathrm{T}}=$ total gene diversity; $H_{\mathrm{S}}=$ gene diversity within populations; $D_{\mathrm{ST}}=$ gene diversity among populations; $G_{\mathrm{ST}}=$ proportion of total gene diversity due to inter- ....1 1

dentity among populations within taxa isideros (below diagonal) (range within paren-

23

\begin{tabular}{|c|c|c|c|c|}
\hline & & $\begin{array}{l}1.938 \\
0.901-0.958)\end{array}$ & & \\
\hline 2. M. tremuloides & 2 & $\begin{array}{l}0.877 \\
(0.831-0.917)\end{array}$ & $\begin{array}{l}0.958 \\
(0.958-0.958)\end{array}$ & \\
\hline 3. M. polymorpha & 9 & $\begin{array}{l}0.874 \\
(0.792-0.968)\end{array}$ & $\begin{array}{l}0.961 \\
(0.904-0.993)\end{array}$ & $\begin{array}{l}0.948 \\
(0.903-0.998)\end{array}$ \\
\hline
\end{tabular}

species but only the pairwise genetic identity for species comparisons are presented in Table 5. The pairwise identities among populations ranged from 0.79 to 1.00 with a mean of 0.92 . Maximum divergence was observed between population 1 ( $M$. rugosa) and populations 8 and 9 (both $M$. polymorpha $)(I=0.79$ and 0.80 respectively). The genetic identities between $M$. rugosa and $M$. tremuloides, $M$. rugosa and $M$. polymorpha, and $M$. tremuloides and $M$. polymorpha were 0.88 , 0.87 , and 0.96 , respectively.

The UPGMA cluster analysis resulted in two discrete groups of populations (Fig. 2). Metrosideros rugosa (1-3) populations segregated from the others at a mean genetic identity of 0.88 . Metrosideros polymorpha populations from Waianae (7-9) were distinct from the remaining populations. The two $M$. tremuloides populations (4-5) were each more similar to $M$. polymorpha populations than they were to each other.

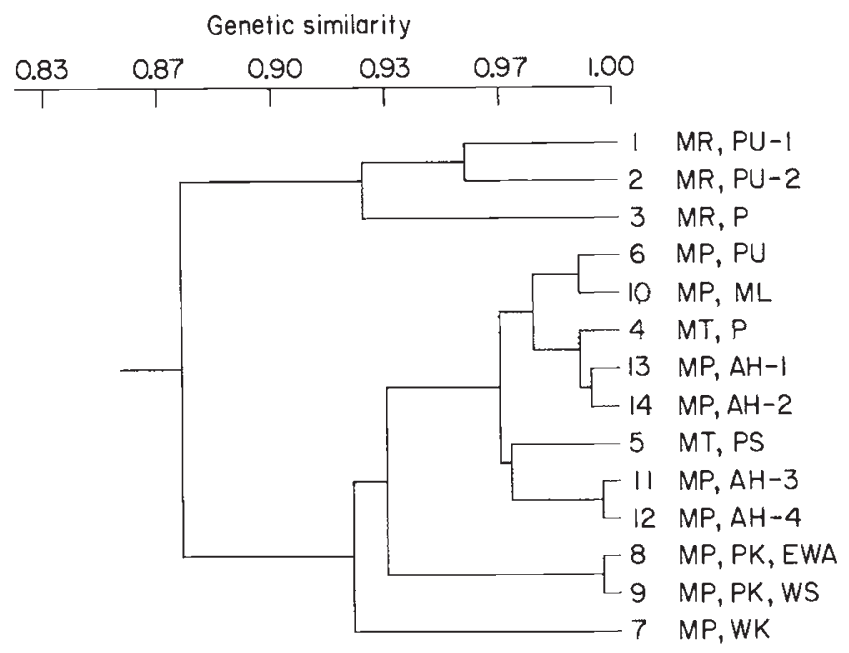

Fig. 2 Dendrogram showing genetic similarities among 14 populations of Metrosideros. 


\section{Discussion}

Hawaiian Metrosideros is an excellent example of a genus that exhibits incipient speciation in an insular environment. The species in this genus may have evolved through local adaptation to various distinctive sites such as bogs, dry and eroded ridge tops and gulches, and similarly, for ecological zones such as lowland dry forests, montane rain forests, cloud forests, and sub-alpine scrub forests on high volcanoes in the islands.

Although there was evidence for differentiation of allele frequencies among the 14 Metrosideros populations for the six loci assessed, the extent of differentiation was marginal. Most populations shared one or two dominant alleles at high frequencies and the unique, low-frequency alleles found in several populations may have originated recently. Similar patterns were frequently observed in outcrossing long-lived trees and woody species (Gottlieb, 1981; Hamrick et al., 1979). Deficiency of heterozygotes observed in some populations of Metrosideros could be due to some degree of self-fertilization.

Little genetic divergence among populations of Metrosideros complex has occurred at the molecular level with 87.7 per cent of the total genetic variation residing within populations. Similar results were obtained in conifer species which occupy large ranges (Guries \& Ledig, 1981; Yeh \& O'Malley, 1981). Hamrick et al. (1979) also demonstrated that plant species, which are widespread, long lived and primarily outcrossed, maintain high levels of intrapopulational genetic variation.

Genetic differentiation appears uniformly low across loci except for Lap-2 and Per. The three taxa involved in the present study showed very little divergence for the isozyme loci studied ( 4.7 per cent) when compared to populations within taxa ( 7.6 per cent).

The foregoing gene diversity analysis of the Hawaiian Metrosideros complex suggests that the taxa in the present study are still in the process of speciation and that the level of isozymic differentiation is indicative of their recent origin. Furthermore, the extremely efficient seed dispersal system, coupled with high rates of outcrossing in the Hawaiian Metrosideros, may act as homogenizing forces to discourage rapid differentiation brought about by disruptive selection. Carlquist (1966) reported that the genetic barriers among island species, especially those which have originated on islands, will be expected to be virtually absent. Hybridization may, as Rattenbury (1962) suggests, help a species survive a 'bottleneck' caused by dwindling land area and climatic stress.
The mean pairwise genetic identity of 0.92 of the 14 populations, including three species, is comparable to values normally obtained for continental conspecific populations (Gottlieb, 1977, 1981; Crawford, 1983). The morphologically distinct $M$. rugosa, however, could be distinguished isozymically from the other two taxa, which suggests that this taxon diverged less recently from $M$. polymorpha than did $M$. tremuloides. The populations of $M$. tremuloides could not be distinguished isozymically from those of $M$. polymorpha, although the two taxa are morphologically discrete. This suggests that the latter two taxa have diverged very recently and their morphological differences for leaf characteristics may be due to small genetic differences not linked to isozyme loci. Metrosideros polymorpha populations from the Waianae mountains have diverged genetically from $M$. polymorpha from the Koolaus, possibly due to distinctness of these two mountains with respect to geological history, age of substrate, climate, and geographical isolation leading to limited gene flow (Fig. 2).

Narrow genetic divergence at isozyme loci among different insular taxa was also reported for Tetramolopium (Lowrey, 1981), Bidens (Ganders \& Nagata, 1984; Helenurm \& Ganders, 1985) and the silversword alliance of species belonging to the genera Argyroxiphium, Dubautia, and Wilkesia (Carr, 1985a,b; Witter \& Carr, 1988) from the Hawaiian islands. Lowrey \& Crawford (1985) found very little isozymic divergence among seven species of Tetramolopium, with a mean genetic identity of 0.95 . Similar results were obtained for six species from two of the three recognized subgenera of the genus Dendroseris which is endemic to the Juan Fernandez Islands, Chile (Crawford et al., 1987a). Hawaiian Bidens with 19 species and eight subspecies endemic to Hawaii, exhibited very little genetic differentiation for isozyme loci (mean $I=0.957$ ) although there is substantial morphological and ecological differentiation (Helenurm \& Ganders, 1985). It has been suggested that congeneric species of insular plants may differ by more conspicuous morphological features than their counterparts on continents in spite of very little genetic divergence (Crawford et al., 1987b). Similarly, Hawaiian Drosophila species show great variation in morphology and behaviour yet exhibit relatively little cytogenetic differentiation (Carson et al., 1970).

These patterns of divergence are similar to those observed in the present study of the genus Metrosideros and appear to be due to the differential adaptive value of morphological versus isozymic or cytogenetic characters. Genetic differentiation at isozyme loci apparently has not occurred at the same rate as that of 
adaptive morphological characters in Hawaiian Metrosideros.

The genetic basis for the morphological differences among different species of Metrosideros may be relatively simple as suggested for other taxa by Hilu (1983), Gottlieb (1984), and Crawford et al. (1987b). Presumably the presence of extensive morphological and genetic variation, coupled with reasonably high levels of heterozygosity, have allowed Hawaiian $M$. polymorpha to function as a tropical lowland, as well as a sub-alpine, canopy tree. Such a range of climatic adaptation is normally exhibited by different species in continental ecosystems (Mueller-Dombois, 1987).

\section{Acknowledgements}

We wish to thank Patricia Welton for assistance in collection of samples in the field and Richard Manshardt and Vincent Lebot for critical discussion of the manuscript. This study was funded through a field study grant awarded by East-West Center.

\section{References}

ARULSEKAR, S. AND PARFITT, D. E. 1986. Isozyme analysis procedures for stone fruits, almonds, grape, walnut, pistachio, and fig. Hort. Sci., 21, 928-933.

ATKINSON, 1. A. E. 1970. Successional trends in the coastal lowland forests of Mauna Loa and Kilauea volcanoes, Hawaii. Pac. Sci., 24, 387-400.

Bousquet, J., CHELIAK, w. M. AND LALONDE, M. 1987. Allozyme variability in natural populations of green alder (Alnus crispa) in Quebec. Genome, 29, 345-352.

BURTON, P. J. 1982. The effect of temperature and light on Metrosideros polymorpha seed germination. Pac. Sci., 36, 229-240.

CARDY, B. J., STUBER, C. W., WENDEL, J. F. AND GOODMAN, M. M. 1983. Techniques for starch gel electrophoresis from maize (Zea mays L.). Mimeograph Series No. 1317, Institute of Statistics, North Carolina State University-Raleigh.

CARLQUIST, s. 1966. The biota of long-distance dispersal. IV. Genetic systems in the floras in the oceanic islands. Evolution, 20, 433-455.

CARPENTER, F. L. 1976. Plant-pollinator interactions in Hawaii: Pollination energetics of Metrosideros collina (Myrtaceae). Ecology, 57, 1125-1144.

CARR, G. D. 1985a. Habitat var ; in the Hawaiian Madiinae (Heliantheae) and its ince to generic concepts in the Compositae. Taxon, 34, 22-25.

CARR, G. D. 1985b. Monograph of the Hawaiian Madiinae (Asteraceae): Argyroxiphium, Dubautia and Wilkesia. Allertonia, 4, 1-123.

CARSON, H. L. 1987. The process whereby species originate. Bioscience, 37, 715-720.

CARSON, H. L., HARDY, D. E., SPIETH, H. T. AND STONE, W. S. 1970. The evolutionary biology of the Hawaiian Drosophilidae. In: Hecht, M. L. and Steere, W. C. (eds), Essays in Evolu- tion and Genetics in Honour of Theodosius Dobzhansky, Appleton Century-Crofts, New York, pp. 437-543.

CORN, C. A. 1972. Seed dispersal methods in Hawaiian Metrosideros. In: Behnke, J. A. (ed.), Challenging Biological Problems: Directions Toward their Solution. Oxford University Press, New York, pp. 422-435.

CORN, C. A. 1979. Variation in Hawaiian Metrosideros. Ph.D. Dissertation. University of Hawaii, Honolulu.

CRAWFORD, D. J. 1983. Phylogenetic and systematic inferences from electrophoretic studies. In: Tanksley, S. O. \& Orton, T. J. (eds), Isozymes in Plant Genetics and Plant Breeding. Elsevier, Amsterdam, pp. 257-287.

CRAWFORD, D. J., STUESSY, T. F. AND SIVA, M. o. 1987a. Allozyme divergence and the evolution of Dendroseris (Compositae: Lactuceae) on the Juan Fernandez Islands. Syst. Bot., 12 , $435-443$

CRAWFORD, D. J., WITKUS, R. AND STUESSY, T. F. 1987b. Plant evolution and speciation on oceanic islands. In: Urbanska, K. M. (ed.), Differentiation Patterns in Higher Plants. Academic Press, New York, pp. 183-199.

GANDERS, F. R. AND NAGATA, K. M. 1984. The role of hybridization in the evolution of Bidens on the Hawaiian Islands. In: Grant, W. F. (ed.), Plant Biosystematics. Academic Press, London, pp. 179-184.

GOTTLEB, L. D. 1977. Electrophoretic data and plant systematics. Ann. Mo. Bot. Gard., 64, 161-180.

GotTLIEB, L. D, 1981. Electrophoretic evidence and plant populations. Progr. Phytochem., 7, 1-46.

GOTTLIEB, L. D. 1984. Genetic and morphological evolution in plants. Am. Natur., 123, 681-709.

GURIES, R. P. AND LEDIG, F. T. 1982. Genetic diversity and population structure in pitch pine (Pinus rigida Mill.). Evolution, 36, 387-402.

HAMRICK, J. L., LINHART, Y. B. AND MITTON, J. B. 1979. Relationships between life history characteristics and electrophoretically detectable genetic variation in plants. Ann. Rev. Ecol. Syst., 10, 173-200.

HELENURM, K. AND GANDERS, F. R. 1985. Adaptive radiation and genetic differentiation in Hawaiian Bidens. Evolution, 39, 753-765.

HiLU, K. W. 1983. The role of single gene mutations in the evolution of flowering plants. Evol. Biol., 16, 97-128.

JAIN, S. K. AND WORKMAN, P. L. 1967. Generalized F-Statistics and the theory of inbreeding and selection. Nature, 214, 674-678.

LOWREY, T. K. 1981. A biosystematic study of Hawaiian Tetramolopium (Compositae:Asteraceae). Ph.D. Dissertation, University of California, Berkeley.

LOWREY, T, K. AND CRAWFORD, D. J. 1985. Allozyme divergence and evolution in Tetramolopium (Compositae:Asteraceae) on the Hawaiian Islands. Syst. Bot., 10, 64-72.

MUELLER-DOMBOIS, D. 1981. Understanding Hawaiian forest ecosystems: the key to biological conservation. In: Mueller-Dombois, D., Bridges, K. W. and Carson, H. L. (eds), Island Ecosystems: Biological Organization in Selected Hawaiian Communities, U.S. IBP Series 15, Hutchinson Ross, Stroudsburg, Pennsylvania.

MUELLER-DOMBOIs, D. 1987. Forest dynamics in Hawaii. Trends Ecol. Evol., 2, 216-220. 
MUELLER-DOMBOIS, D. AND LOOPE, L. L. 1990. Some unique ecological aspects of oceanic island ecosystems. Monogr. Syst. Bot. Missouri Bot. Gard., 32, 21-27.

NEI, M. 1973. Analysis of gene diversity in subdivided populations. Proc. Nat. Acad. Sci., USA, 70, 3321-3323.

NEI, M. 1978. Estimation of average heterozygosity and genetic distance from a small number of individuals. Genetics, 89, 583-590.

PORTER, J. R. 1972. The Growth and Phenology of Metrosideros in Hawaii. Ph.D. Dissertation, University of Hawaii, Honolulu.

RATTEnbury, J. A. 1962. Cyclic hybridization as a survival mechanism in the New Zealand forest flora. Evolution, 16, 348-363.

Rock, J. F. 1917. The Ohia Lehua trees of Hawaii. Board of Agriculture and Forestry Botanical Bulletin No. 4, Honolulu.

SHAW, C. R. AND PRASAD, R. 1970. Starch gel electrophoresis of enzymes - a compilation of recipes. Biochem. Genet., 4, 297-320.

SKOTTSBERG, C. 1944. Hawaiian vascular plants. IV. Meddelanden fran Goteborgs Botaniska Tradgard., 15, 402-409.

SMITH, A. C. 1973. Studies of Pacific Islands plants. XXVI. Metrosideros collina (Myrtaceae) and its relatives in the southern Pacific. Am. J. Bot., 60, 479-490.

SNEATH, P. H. A. AND SOKAL, R. R, 1973. Numerical Taxonomy. W. H. Freeman, San Francisco.

STEMMERMANN, L. 1983. Ecological studies of Hawaiian Metrosideros in a successional context. Pac. Sci., 37, 361-373.

SWOFFORD, D. L. AND SELANDER, R. B. 1989. BIOSYS-1 a computer program for the analysis of allelic variation in population genetics and biochemical systematics. Illinois Natural History Survey, Illinois.

SYSTMA, K. J. AND SCHAAL, B. A. 1985. Genetic variation, differentiation, and evolution in a species complex of tropical shrubs based on isozymic data. Evolution, 39, 582-593.

WITTER, M. S. AND CARR, G. D. 1988. Adaptive radiation and genetic differentiation in the Hawaiian Silversword alliance (Compositae:Madiinae). Evolution, 42, 12781287.

WRIGHT, s. 1965 . The interpretation of population structure by F-statistics with special regard to systems of mating. Evolution, 19, 395-420.

YEH, F. AND O'MALLEY, D. M. 1981. Enzyme variation in natural populations of Douglas-fir (Pseudotsuga menziesii (Mirb.) Franco) from British Columbia. I. Genetic variation patterns in coastal populations. Silvae Genet., 29, 83-92. 